\title{
Langmuir
}

pubs.acs.org/Langmuir

(C) 2010 American Chemical Society

\section{ssDNA Binding Reveals the Atomic Structure of Graphene}

\author{
By Sudhir Husale, ${ }^{\S, \dagger}$ Sangeeta Sahoo, ${ }^{\S}$, Aleksandra Radenovic, ${ }^{*}, \dagger$ Floriano Traversi, ${ }^{\dagger}$ \\ Paolo Annibale, ${ }^{\dagger}$ and Andras Kis
}

\begin{abstract}
${ }^{\dagger}$ Laboratory of Nanoscale Biology, Institute of Bioengineering, School of Engineering, EPFL, 1015 Lausanne, Switzerland, and ${ }^{\star}$ Laboratory of Nanoscale Electronics and Structures Institute of Electrical Engineering, School of Engineering, EPFL, 1015 Lausanne, Switzerland. ${ }^{\S}$ These authors contributed equally to this work
\end{abstract}

Received June 21, 2010. Revised Manuscript Received October 8, 2010

\begin{abstract}
We used AFM to investigate the interaction of polyelectrolytes such as ssDNA and dsDNA molecules with graphene as a substrate. Graphene is an appropriate substrate due to its planarity, relatively large surfaces that are detectable via an optical microscope, and straightforward identification of the number of layers. We observe that in the absence of the screening ions deposited ssDNA will bind only to the graphene and not to the $\mathrm{SiO}_{2}$ substrate, confirming that the binding energy is mainly due to the $\pi-\pi$ stacking interaction. Furthermore, deposited ssDNA will map the graphene underlying structure. We also quantify the $\pi-\pi$ stacking interaction by correlating the amount of deposited DNA with the graphene layer thickness. Our findings agree with reported electrostatic force microscopy (EFM) measurements. Finally, we inspected the suitability of using a graphene as a substrate for DNA origami-based nanostructures.
\end{abstract}

\section{Introduction}

Graphene is a single layer of two-dimensional $\mathrm{sp}^{2}$ carbon atoms that are closely packed into a honeycomb structure. This new kind of carbon-based nanostructured material was first produced in $2004,{ }^{1}$ and since then it has attracted a great deal of attention due to its exceptional electrical, ${ }^{2,3}$ mechanical, ${ }^{4-7}$ and thermal properties. $^{8}$ These properties make graphene the ideal substrate for future hybrid organic-inorganic nanostructures.

Hybrid organic-inorganic nanostructures are appealing due to their ability to combine the best properties of the two components. Among organic molecules, exceptional graphene properties could be matched the best by DNA molecule which has following properties: hybridization, convenient synthesis of designed sequences, and its widespread application in structural DNA nanotechnology. ${ }^{9-11}$ The listed DNA properties contributed to its wide use as a building block of much hybrid

*Corresponding author. E-mail: aleksandra.radenovic@epfl.ch.

(1) Novoselov, K. S.; Geim, A. K.; Morozov, S. V.; Jiang, D.; Zhang, Y.; Dubonos, S. V.; Grigorieva, I. V.; Firsov, A. A. Science 2004, 306(5296), 666-669.

(2) Novoselov, K. S.; Geim, A. K.; Morozov, S. V.; Jiang, D.; Katsnelson, M. I.; Grigorieva, I. V.; Dubonos, S. V.; Firsov, A. A. Nature 2005, 438(7065), 197-200.

(3) Zhang, Y. B.; Tan, Y. W.; Stormer, H. L.; Kim, P. Nature 2005, 438(7065), 201-204.

(4) Verbridge, S. S.; Craighead, H. G.; Parpia, J. M. Appl. Phys. Lett. 2008, 92 (1), 013112.

(5) Meyer, J. C.; Geim, A. K.; Katsnelson, M. I.; Novoselov, K. S.; Booth, T. J.; Roth, S. Nature 2007, 446(7131), 60-63.

(6) Meyer, J. C.; Geim, A. K.; Katsnelson, M. I.; Novoselov, K. S.; Obergfell, D.; Roth, S.; Girit, C.; Zettl, A. Solid State Commun. 2007, 143(1-2), 101-109.

(7) Bunch, J. S.; van der Zande, A. M.; Verbridge, S. S.; Frank, I. W.; Tanenbaum, D. M.; Parpia, J. M.; Craighead, H. G.; McEuen, P. L. Science 2007, 315(5811), 490-493.

(8) Balandin, A. A.; Ghosh, S.; Bao, W. Z.; Calizo, I.; Teweldebrhan, D.; Miao, F.; Lau, C. N. Nano Lett. 2008, 8(3), 902-907.

(9) Douglas, S. M.; Dietz, H.; Liedl, T.; Hogberg, B.; Graf, F.; Shih, W. M. Nature 2009, 459(7245), 414-418.

(10) Andersen, E. S.; Dong, M.; Nielsen, M. M.; Jahn, K.; Subramani, R.; Mamdouh, W.; Golas, M. M.; Sander, B.; Stark, H.; Oliveira, C. L. P.; Pedersen, J. S.; Birkedal, V.; Besenbacher, F.; Gothelf, K. V.; Kjems, J. Nature 2009, 459 (7243), 73-U75.

(11) Rothemund, P. W. K. Nature 2006, 440(7082), 297-302.

(12) Katz, E.; Willner, I. ChemPhys Chem 2004, 5(8), 1085-1104.

(13) Hess, H.; Bachand, G. D.; Vogel, V. Chem.-Eur. J. 2004, 10(9), 2110-2116.

(14) Kim, S. J.; Kim, B. H. Nucleic Acids Res. 2003, 31(11), 2725-2734.

(15) Niemeyer, C. M. Chem.-Eur. J. 2001, 7(15), 3189-3195. nanostructure. ${ }^{12-18}$ For example, ssDNA-carbon nanotube (ssDNA-CNT) hybrids present a new form of hybrid nanostructure. This novel hybrid nanomaterial consists of single-walled carbon nanotubes (CNT) wrapped with a self-assembled coating of single-stranded DNA (ssDNA), and it has unique properties that derive from these two components. ssDNA-CNT have been used for the following applications: CNT solubilization, ${ }^{19}$ sorting, ${ }^{20}$ and patterned placement of nanotubes. ${ }^{21}$

However, mechanisms of the DNA-based solubilization and separations are still poorly understood. ${ }^{22}$ The structural information comes primarily from low-resolution atomic force microcopy (AFM) taken on dried ssDNA/CNT samples. ${ }^{23}$ So far, obtained experimental evidence suggests that the hybrid structure is dependent on both DNA sequence and CNT structure.

AFM imaging of ssDNA wrapped around CNT is extremely challenging; thus, we hypothesized that the AFM imaging of selfassembled monolayers of ssDNAs on the graphene could provide novel insights into strength, nature, and mechanism for their interaction. Graphene represents the closest model system to the $\mathrm{CNT}$, and it is commonly used in theoretical studies as a substitute for CNTs. ${ }^{22}$ Its planarity permits more detailed examination of its interactions with polyelectrolytes such as ssDNA and dsDNA by AFM. Current theoretical studies suggest that the nucleobases exhibit significantly different interaction strengths when physisorbed on graphene. ${ }^{22}$ Although the magnitude of interaction varies for the four nitrogenous DNA bases, ${ }^{22}$ it is still strong enough to

(16) Niemeyer, C. M.; Ceyhan, B.; Gao, S.; Chi, L.; Peschel, S.; Simon, U. Colloid Polym. Sci. 2001, 279(1), 68-72.

(17) Niemeyer, C. M.; Ceyhan, B.; Blohm, D. Bioconjugate Chem. 1999, 10(5), 708-719.

(18) Cassell, A. M.; Scrivens, W. A.; Tour, J. M. Angew. Chem., Int. Ed. 1998, 37 (11), 1528-1531.

(19) Zheng, M.; Jagota, A.; Semke, E. D.; Diner, B. A.; Mclean, R. S.; Lustig, S. R.; Richardson, R. E.; Tassi, N. G. Nature Mater. 2003, 2(5), 338-342.

(20) Zheng, M.; Jagota, A.; Strano, M. S.; Santos, A. P.; Barone, P.; Chou, S. G.; Diner, B. A.; Dresselhaus, M. S.; McLean, R. S.; Onoa, G. B.; Samsonidze, G. G.; Semke, E. D.; Usrey, M.; Walls, D. J. Science 2003, 302(5650), 1545-1548. (21) McLean, R. S.; Huang, X. Y.; Khripin, C.; Jagota, A.; Zheng, M. Nano Lett. 2006, 6(1), 55-60.

(22) Gowtham, S.; Scheicher, R. H.; Ahuja, R.; Pandey, R.; Karna, S. P. Phys. Rev. B 2007, 76(3), 033401

(23) Tu, X. M.; Zheng, M. Nano Res. 2008, 1(3), 185-194. 

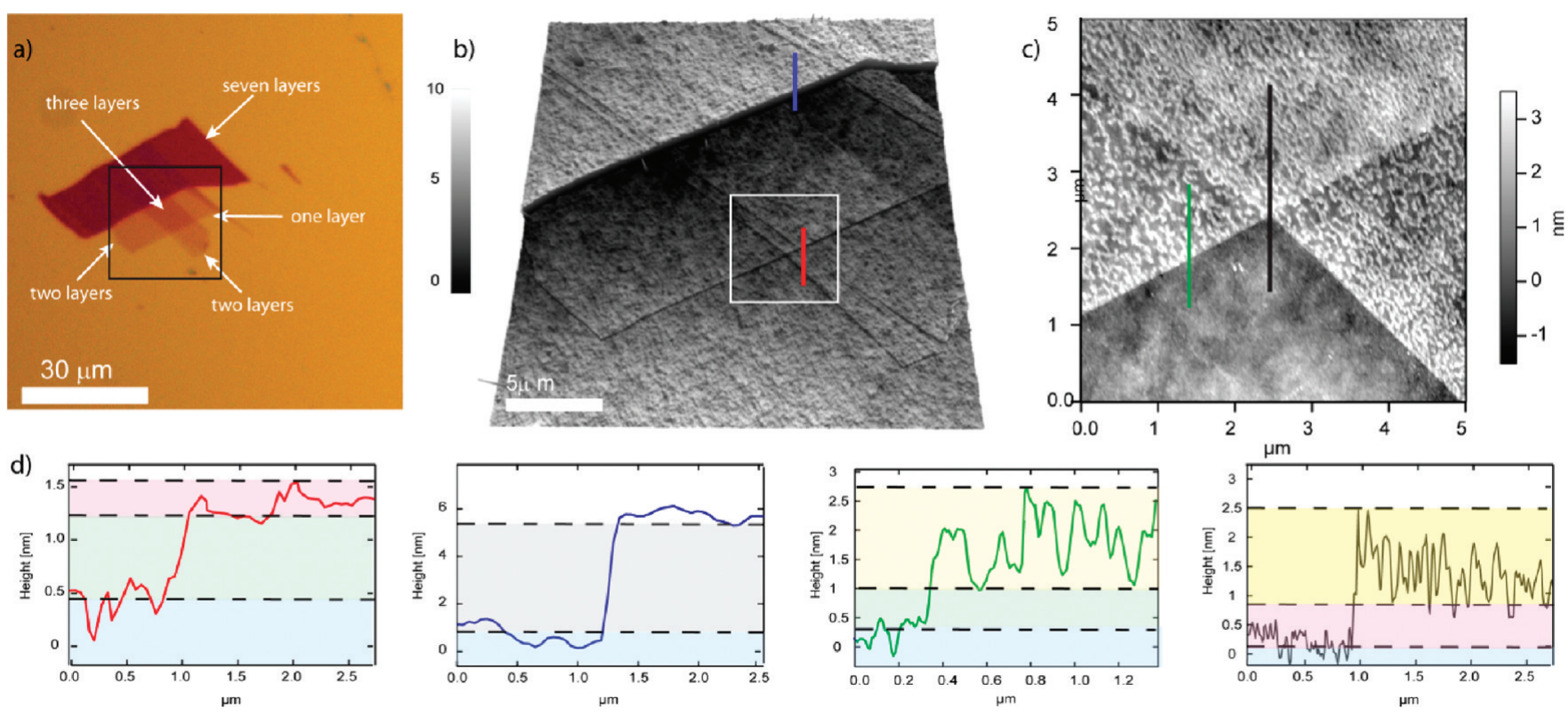

Figure 1. (a) Optical image of a graphene sample deposited on an $\mathrm{SiO}_{2}$ substrate. Number of layers determined from optical contrast and confirmed using AFM. (The scale bar is $30 \mu \mathrm{m}$.) (b) An expanded 3D AFM image of the boxed region in (a) prior to ssDNA deposition. (The scale bar is $5 \mu \mathrm{m}$.) (c) An expanded AFM image of the boxed region in (b) after ssDNA deposition. (d) Corresponding color-coded cross sections.

assemble ssDNA on a graphite surface into a monolayer through hydrogen-bonding interactions mapping the underlying lattice structure. Recent molecular dynamics simulations suggest that the absorbed ssDNA chains maximize stacking by adopting conformations in which bases alternate from one side to the backbone. ${ }^{24}$

In order to test these theoretical results, we performed AFM imaging of ssDNA directly deposited on graphene. The ssDNA-graphene hybrid system was also recently used to facilitate direct exfoliation from graphite flakes and further hybridization with a gold nanoparticle labeled with cDNA. ${ }^{25}$ The presented work demonstrates first appealing applications of this novel hybrid system.

In addition, we examined the suitability of graphene substrate for future electrochemical DNA sensors that would utilize twodimensional origami templates. In this novel hybrid system graphene could be used as the electrical interconnect and as an e-beam tailored substrate, while origami can be used as template for material growth, ${ }^{26,27}$ sensor, ${ }^{28,29}$ or even as a template for specific single-molecule chemical reactions. ${ }^{30}$

\section{Results}

We have obtained variable numbers of graphene layers by the standard method of mechanical exfoliation of $\mathrm{HOPG}^{31}$ on

(24) Tu, X. M.; Manohar, S.; Jagota, A.; Zheng, M. Nature 2009, 460(7252), $250-253$

(25) Liu, F.; Choi, J. Y.; Seo, T. S. Chem. Commun. 2010, 46(16), 2844-2846.

(26) Ding, B. Q.; Deng, Z. T.; Yan, H.; Cabrini, S.; Zuckermann, R. N.; Bokor, J. J. Am. Chem. Soc. 2010, 132(10), 3248-+.

(27) Maune, H. T.; Han, S. P.; Barish, R. D.; Bockrath, M.; Goddard, W. A Rothemund, P. W. K.; Winfree, E. Nature Nanotechnol. 2010, 5(1), 61-66.

(28) Modi, S.; Swetha, M. G.; Goswami, D.; Gupta, G. D.; Mayor, S.; Krishnan, Y. Nature Nanotechnol. 2009, 4(5), 325-330.

(29) Stoliar, P.; Bystrenova, E.; Quiroga, S. D.; Annibale, P.; Facchini, M.; Spijkman, M.; Setayesh, S.; de Leeuw, D.; Biscarini, F. Biosens. Bioelectron. 2009, 24(9), 2935-8.

(30) Voigt, N. V.; Torring, T.; Rotaru, A.; Jacobsen, M. F.; Ravnsbaek, J. B.; Subramani, R.; Mamdouh, W.; Kjems, J.; Mokhir, A.; Besenbacher, F.; Gothelf, K. V. Nature Nanotechnol. 2010, 5(3), 200-203.

(31) Novoselov, K. S.; Jiang, D.; Schedin, F.; Booth, T. J.; Khotkevich, V. V.; Morozov, S. V.; Geim, A. K. Proc. Natl. Acad. Sci. U.S.A. 2005, 102(30), 1045110453. lithographically patterned $\mathrm{SiO}_{2}$ substrates (for details on substrate preparation see Supporting Information). Identification and determination of the number of graphene layers have been performed by optical microscopy. Well-established experimental protocols $^{32-34}$ that rely on thin film interference can be used to easily determine the number of layers in a given graphene flake from the optical contrast.

As an additional verification step, we also performed AFM imaging of graphene deposited onto $\mathrm{SiO}_{2}$ substrates prior to the DNA deposition. As already observed, AFM-based measurements of graphene thickness can vary from 0.35 to $1.4 \mathrm{~nm}$ relative to the $\mathrm{SiO}_{2}$ substrate ${ }^{33-35}$ depending on the measurement conditions (scanning parameters), sample preparation procedures, and other laboratory conditions (for example humidity). We chose overlapping of folded graphene regions for the most reliable thickness measurements (see Figure 1). Upon AFM imaging of the pristine graphene surfaces and after establishing the number of graphene layers, we proceeded with ssDNA/dsDNA deposition.

To obtain ssDNA, Lambda DNA (purchased from NEB) was heated at $90{ }^{\circ} \mathrm{C}$ for 10 min prior to deposition. All DNA preparations were diluted either in nanopure (Ultra High Quality Millipore) water or in $1 \mathrm{mM}$ Tris- $\mathrm{HCl}$ buffer to a final DNA concentration of $5 \mu \mathrm{g} / \mathrm{mL}$.

A $50 \mu \mathrm{L}$ aliquot of the DNA solution was deposited onto the substrate and incubated for $20 \mathrm{~min}$ at room temperature, and then the sample was rinsed with nanopure water and dried with nitrogen. Next, we performed tapping mode AFM imaging (for details on AFM imaging see Supporting Information) in order to confirm the adsorption of ssDNA molecules on the graphene substrate. Typically, we observe relative increase in height of

(32) Jung, I.; Pelton, M.; Piner, R.; Dikin, D. A.; Stankovich, S.; Watcharotone, S.; Hausner, M.; Ruoff, R. S. Nano Lett. 2007, 7(12), 3569-3575.

(33) Casiraghi, C.; Hartschuh, A.; Lidorikis, E.; Qian, H.; Harutyunyan, H.; Gokus, T.; Novoselov, K. S.; Ferrari, A. C. Nano Lett. 2007, 7(9), 2711-2717.

(34) Blake, P.; Hill, E. W.; Neto, A. H. C.; Novoselov, K. S.; Jiang, D.; Yang, R.; Booth, T. J.; Geim, A. K. Appl. Phys. Lett. 2007, 91(6), 063124.

(35) Chen, Z. H.; Lin, Y. M.; Rooks, M. J.; Avouris, P. Physica E 2007, 40(2), 228-232. 
$1 \pm 0.2 \mathrm{~nm}$ in the topographic profile upon ssDNA deposition and $2.1 \pm 0.2 \mathrm{~nm}$ upon dsDNA deposition.

If ssDNA deposition is performed in pure water, it will bind exclusively to the pristine graphene surface as shown in Figure 1c. Remarkably, ssDNA substrate selectivity originates from the nature of the ssDNA/graphene binding energy that is largely due to the $\pi-\pi$ stacking interaction, but it also has contributions from the sugar-phosphate backbone. ${ }^{36}$ The observed selectivity will be gradually suppressed if the ssDNA deposition is performed in electrolyte solutions of varying $\mathrm{Mg}^{2+}$ ionic strength.

We observed that ssDNA molecules adsorbed to graphene adopt a wealth of secondary structures shown in Figure 1, which originated partially from intrastrand base pairing and partially from the strong interaction of DNA bases with graphene (binding energy of $\sim 10 k T$ per nucleotide or larger with slight differences between the four nucleotides ${ }^{23}$ ). In addition, we noticed that the

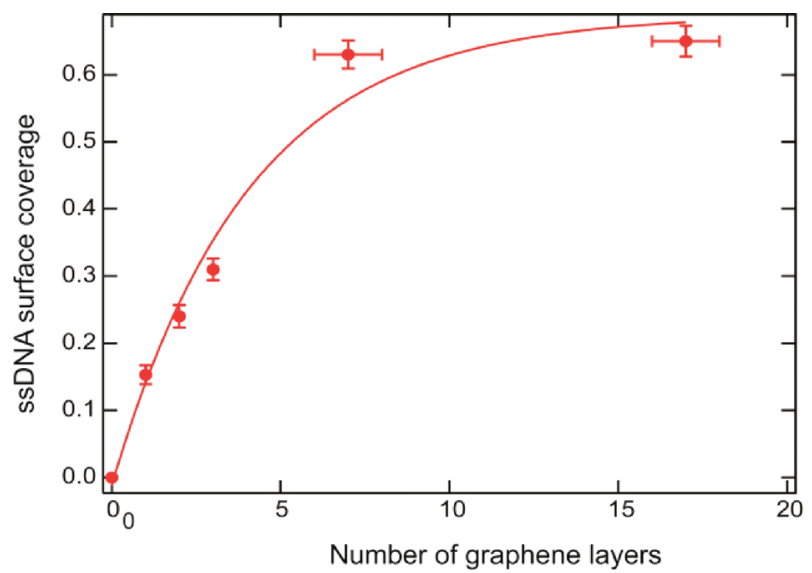

Figure 2. ssDNA surface coverage as a function of the number of layers. Data points for 1, 2, 3, 7 \pm 1 , etc., layers are averages of data from three different regions of equal layer thickness. Vertical error bars represent the standard error, and horizontal error bars represent the uncertainty in layer thickness measurements.
ssDNA binding efficiency correlates with the number of pristine graphene layers. Similar behavior has been recently reported by Mohanty et al. $^{37}$ The authors report on a higher fluorescence intensity in thicker regions of functionalized graphene oxide (GO) upon hybridization with fluorescently labeled complementary strands, indicating that the short ssDNA preferentially binds on thicker layers (including folds) and wrinkles. Even though GO has been functionalized with carboxyl groups, GO layers of different thickness have different surface potential, ${ }^{38}$ resulting in a variation of the DNA density. This variation in surface potential is due to a difference in the magnitude of intrinsic screening of interfacial traps or defects present on the substrate. Local field enhancement at the sharp edges of wrinkles is screened via uniform distribution of carboxylic acid groups.

AFM imaging of long ssDNA molecules deposited on pristine graphene can efficiently probe surface at different layers of thickness and further quantify the interaction strength. As shown in Figures 1c and 3, ssDNA coverage indeed correlates with the number of layers, and higher DNA coverage of wrinkles can be attributed to the local field enhancement at the sharp edges of wrinkles. The nonbound carbon atoms existing on these defects may form bonds with hydrogen, hydroxyl, and carboxyl groups. To quantify and evaluate ssDNA binding efficiency as a function of the number of graphene layers, we performed AFM imaging and analysis of $1 \mu \mathrm{m}^{2}$ areas at different layers of thickness (Figure 2). We observed saturation in the ssDNA surface coverage at $7 \pm 1$ layers, indicating that surface potential increases with film thickness and quickly approaches to the bulk value. Our results agree with reported electrostatic force microscopy (EFM) measurements. $^{38}$

Even more striking is how well ssDNA molecules can map the underlying graphene lattice structure. Preferred ssDNA orientations, as shown in Figure 1c, Figure 3, and Supporting Information Figure 2 are easily identified. Enhanced ssDNA binding to edges and wrinkles allows fast image segmentation. For example, the AFM image shown in Figure 3 has been segmented into four regions (no DNA, and regions 1, 2, and 3 with three preferred ssDNA orientations).
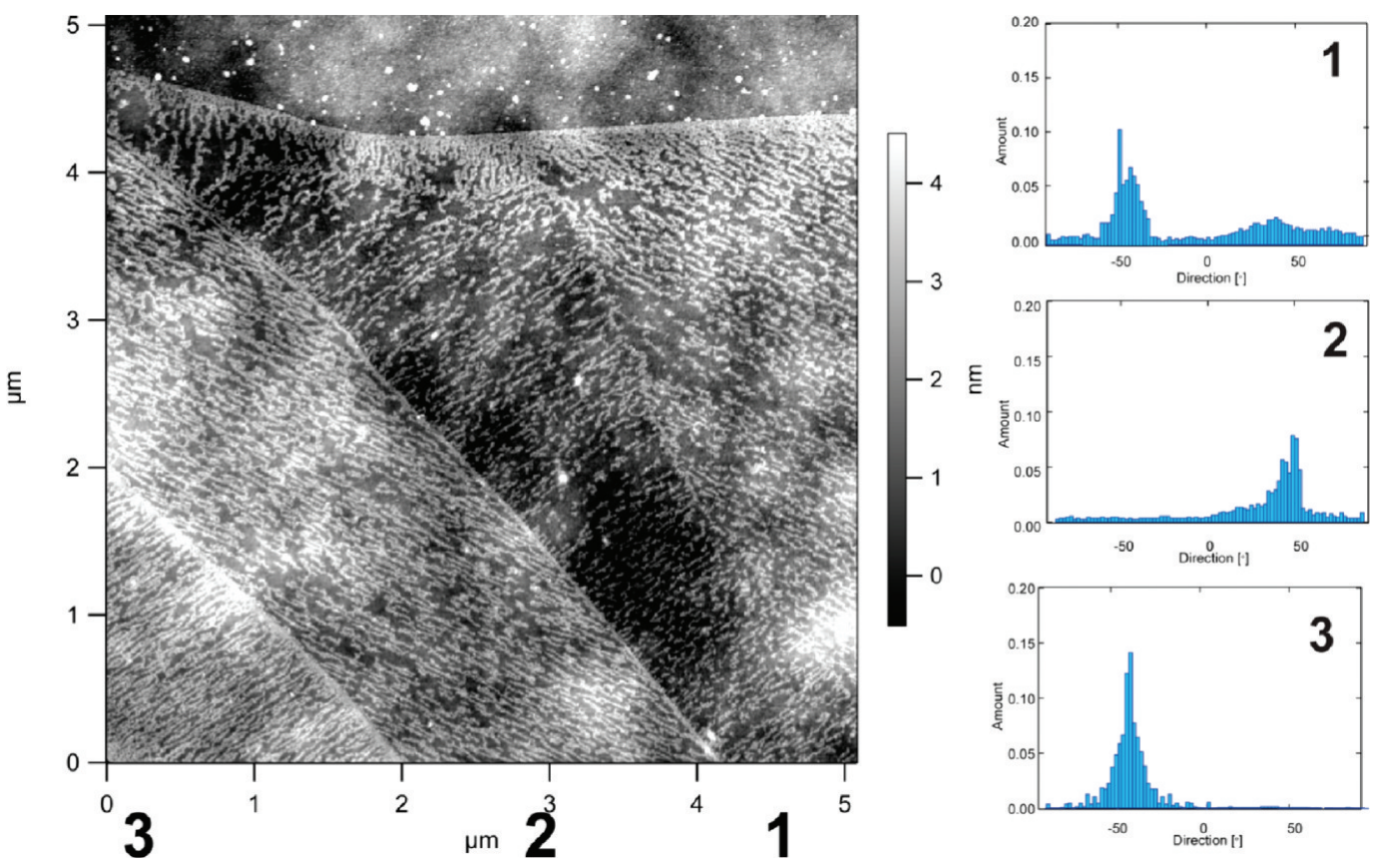

Figure 3. AFM image of lambda ssDNA adsorbed on the pristine graphene deposited on $\mathrm{SiO}_{2}$ substrate. Histograms of orientations obtained form three regions numerated in the AFM image. 
a)

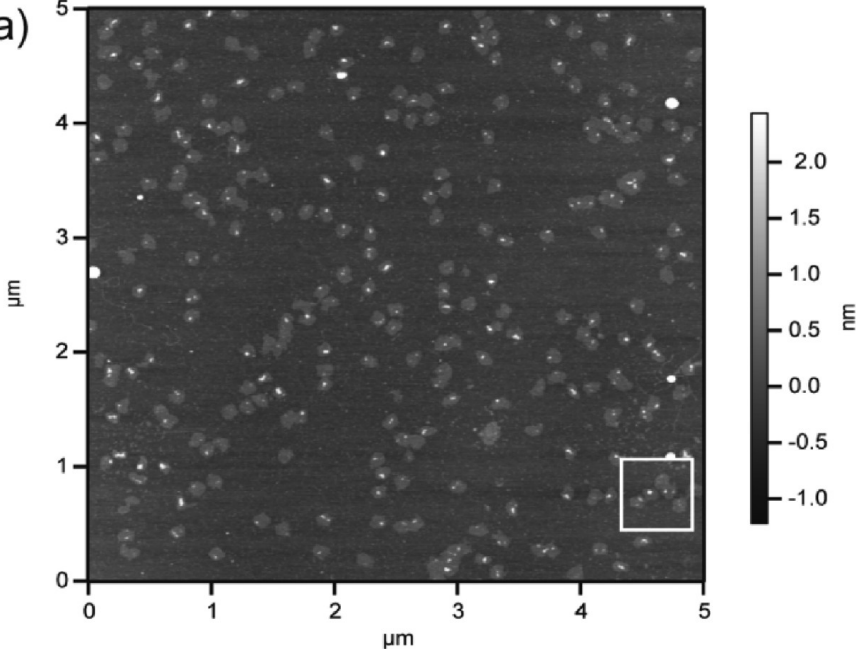

c)

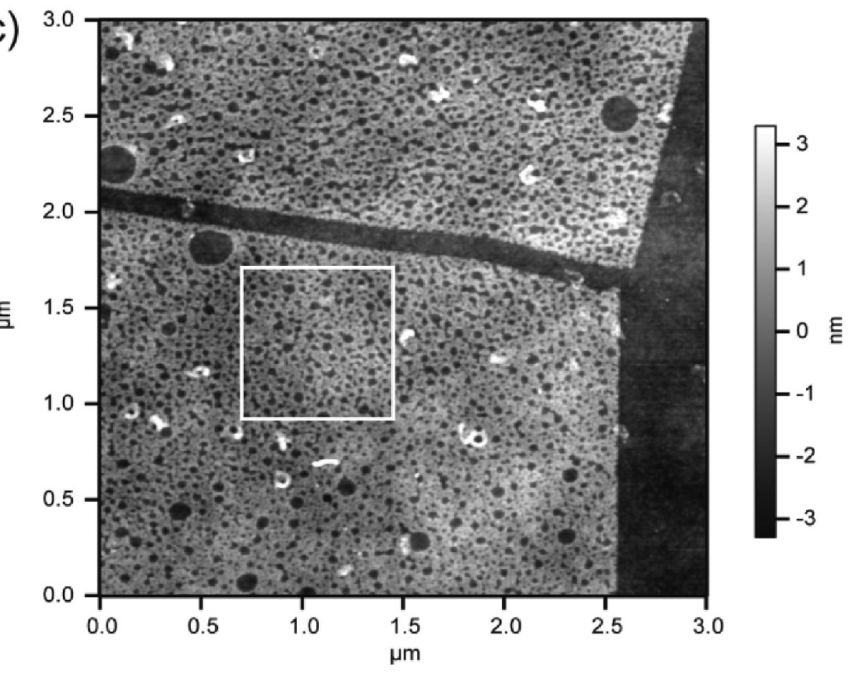

b)

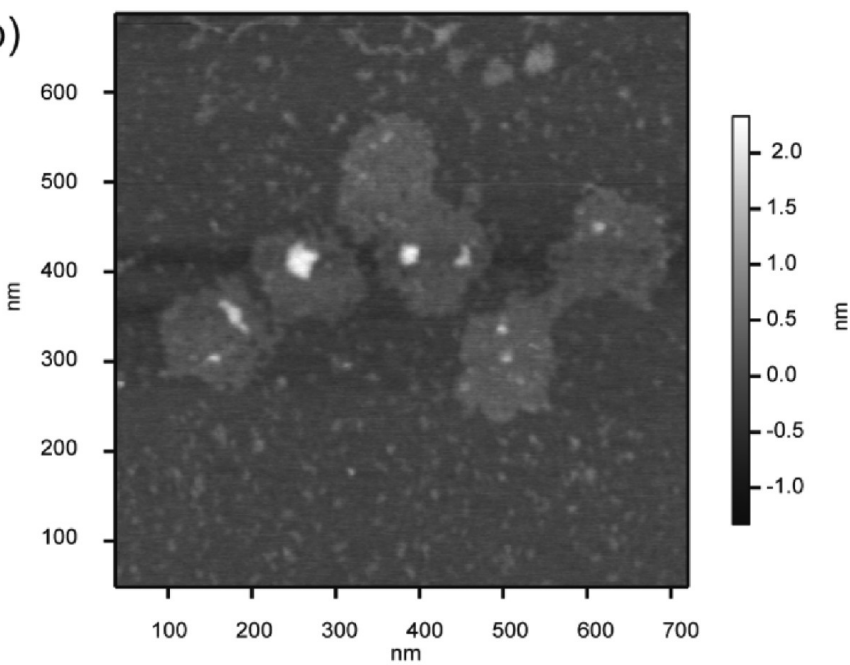

d)

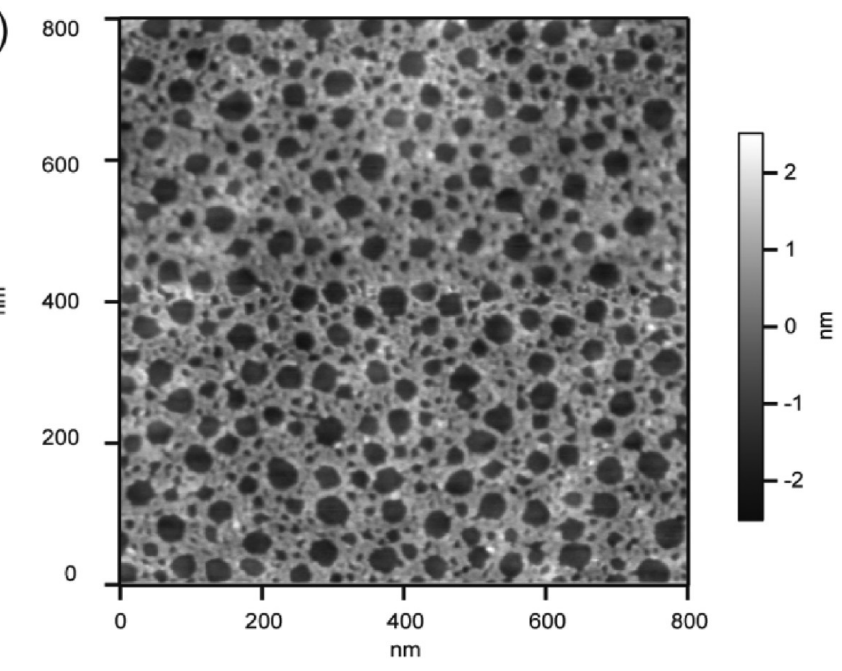

Figure 4. (a) AFM image of a DNA origami adsorbed on the mica surface. (b) An expanded AFM image of the boxed region in (a). (c) AFM image of a DNA origami adsorbed on the pristine graphene deposited on $\mathrm{SiO}_{2}$ substrate. (d) An expanded AFM image of the boxed region in (c). Depositions were performed either in $10 \mathrm{mM}$ Tris- $\mathrm{HCl}$; buffer solution containing $12 \mathrm{mM} \mathrm{Mg}^{2+}$ ions (a, b) or ultrapure water (c, d).

To quantify the preferred orientation of structures present in the image, we have used ImageJ with the Fiji Directionality plugin. It computes a histogram indicating the amount of structures in a given direction. Images with completely isotropic content are expected to give a flat histogram (see Supporting Information Figure 1), whereas images in which there is a preferred orientation are expected to give a histogram with a peak at the preferred orientation, as shown in histograms in Figure 3. Depending on the degree of folding, many more preferred ssDNA orientations can be identified. For example, in Supporting Information Figure 2 we have identified six preferred orientations on a structure with several overlapping graphene layers. Besides low ionic strength, the graphene cleanliness is the second important parameter in the ssDNA orientation on the pristine graphene.

Intrigued by the possibility to orient ssDNA molecules on the substrate such as graphene, we have designed several DNA origami structures $^{11}$ with variable ssDNA content $(18 \%$ and $36 \%$; see Supporting Information). Magnesium chloride $\left(\mathrm{MgCl}_{2}\right)$ was added to the DNA solution containing origami structures to a final concentration of $12 \mathrm{mM}$ in order to stabilize

(36) Wang, Y. J. Phys. Chem. C 2008, 112(37), 14297-14305.

(37) Mohanty, N.; Berry, V. Nano Lett. 2008, 8(12), 4469-4476.

(38) Datta, S. S.; Strachan, D. R.; Mele, E. J.; Johnson, A. T. C. Nano Lett. 2009, 9(1), 7-11 folded structures. To examine if the origami structures folded properly, the solution containing the origami was deposited on the freshly cleaved mica substrate. The formation of squares and rectangles is confirmed by imaging of the assembled origami structures on mica surface (see Figure 4). After establishing that our solution contained the desired DNA origami shapes, we diluted the solution containing DNA origami in ultrapure water to minimize the ionic strength of the folding buffer prior to deposition on graphene. Unfortunately, the origami folding buffer contains magnesium $\left(\mathrm{Mg}^{2+}\right)(12 \mathrm{mM})$ ions that neutralize negative charges on the DNA and allowed the single-stranded DNA to come together and form a double helix or DNA origami.

If diluted in ultrapure water, the origami structures become unstable. Still, the deposited origami covered only the graphene surface, but they lost their folded structure. If the deposition is performed in the presence of $\mathrm{Mg}^{2+}$ ions, structures are deposited on the entire substrate without specificity for the graphene. Furthermore, the presence of the $\mathrm{Mg}^{2+}$ ions weakened the graphene adhesion to the $\mathrm{SiO}_{2}$ substrate and caused detachment of most of the flakes.

We assumed that the ability of ssDNA to map the underlying lattice structure through bonding interactions would be influenced by the presence of screening ions. ${ }^{39}$ In order to test this

(39) Manohar, S.; Tang, T.; Jagota, A. J. Phys. Chem. C 2007, 111(48), 1783517845 . 

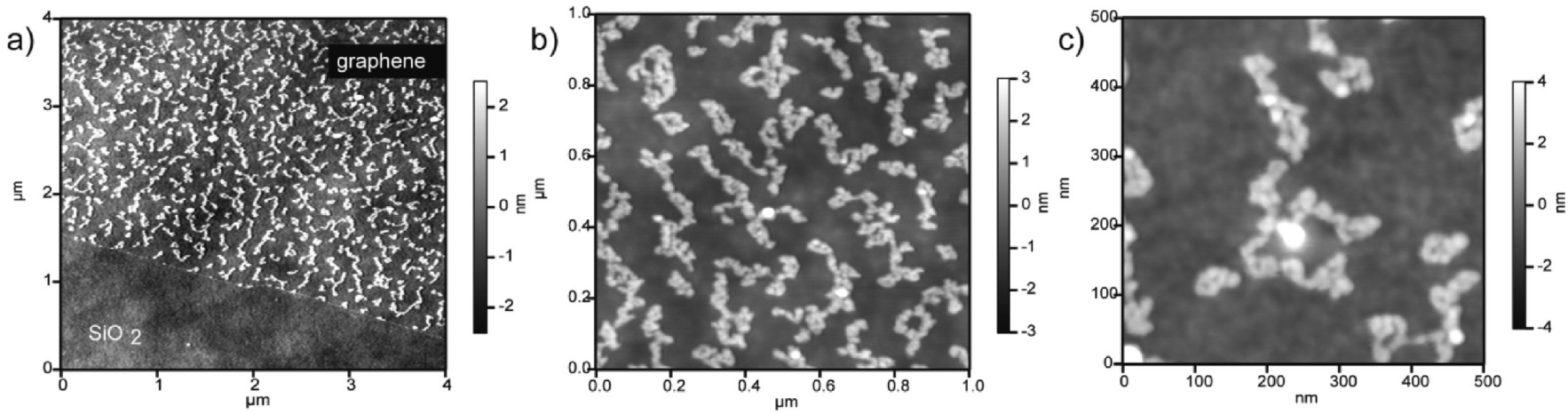

Figure 5. $(\mathrm{a}-\mathrm{c}) \mathrm{AFM}$ images at different magnifications of ssDNA are adsorbed on the pristine graphene deposited on $\mathrm{SiO}_{2}$ substrate Deposition were performed either in Tris- $\mathrm{HCl}$; buffer solution containing $5 \mathrm{mM} \mathrm{Mg}{ }^{2+}$ ions. Deposited ssDNAs molecules display no detectable directionality.

assumption, we deposited ssDNA in the presence of ions $(1 \mathrm{mM}$ Tris- $\mathrm{HCl}$ buffer) and examined the sample using AFM. On the AFM images (for example see Figure 5), preferred ssDNA orientation was not detected either by eye or the use of Fiji Directionality plug-in. However, ssDNA still binds preferentially to the graphene surface.

Keeping the same deposition conditions, we deposited the mixture of ssDNA and dsDNA. In Supporting Information Figure 3 several dsDNA molecules is clearly distinguished among the wealth of structures adopted by ssDNA molecules, again without proffered orientation. When the interaction between dsDNA and the substrate is strong, dsDNA will be quenched on the surface, and there will be no equilibration in two dimensions. Furthermore, the obtained AFM images will represent some form of a two-dimensional projection of their three-dimensional bulk conformations as shown in ref 40 . In our case, lambda DNA cohesive ends, 12 base long single-stranded overhangs, interact through $\pi-\pi$ stacking with the graphene flakes, while the rest of the dsDNA interaction with the graphene might be only due to the weak van der Waals. Because of the different interaction strengths, between binding points of lambda DNA cohesive ends and the rest of the dsDNA we obtain increased end-to-end distance of adsorbed dsDNA molecules.

\section{Discussion}

In summary, we have investigated the binding of ssDNA on graphene. In the absence of the screening ions, ssDNA will bind exclusively to graphene and apparently map its lattice orientation.

What could be a physical mechanism that governs observed ssDNA alignment on graphene? Although the answer is not completely clear to us at the moment, we do wish to propose possible mechanism: First in the given graphene region we observe mostly one ssDNA orientation and not the other two that are expected since graphene's lattice repeats itself every $60^{\circ}$. We hypothesize that the edge states and surface defects might serve as nucleation centers that favor one of orientation over the

(40) Valle, F.; Favre, M.; De Los Rios, P.; Rosa, A.; Dietler, G. Phys. Rev. Lett. 2005, 95(15), 158105 . other two. Next, as has been shown in ref 24, the absorbed energyminimized 2D DNA sheet on the graphene layer will be composed of the aligned strands.

The observed DNA binding specificity is retained in the low salt buffers, and it might be combined with recent advances in fabrication and handling of graphene-based biosensors ${ }^{41-43}$ in order to improve their sensitivity and selectivity.

The observed binding selectivity combined with the graphene transparency and high conductive surface area that can be lithographically patterned provides new opportunities for the graphene integration into biosensor arrays. As a transducer in future DNA biosensors, graphene can be used without prior functionalization.

Our measurements show that the binding density of DNA on graphene is dependent on the number of graphene layers. In addition, we observed saturation in the ssDNA surface coverage at $7 \pm 1$ layers, closely matched by previous observations on the dependence of surface potential. ${ }^{38}$

Finally, we investigated the role of ions in the binding process. Our preliminary study indicates the importance of screening ions in the interaction between graphene and ssDNA while our results on graphene and DNA origami may be exploited to construct DNA-graphene nanobiosensors.

Acknowledgment. This work was supported by FP7 nano DNA sequencing grant and Swiss National Science Foundation (FNS) grant 200021-125319.

Supporting Information Available: Preparation of substrates for AFM, AFM experimental conditions, DNA origami synthesis, and additional AFM figures. This material is available free of charge via the Internet at http://pubs. acs.org.

(41) Shan, C. S.; Yang, H. F.; Song, J. F.; Han, D. X.; Ivaska, A.; Niu, L. Anal. Chem. 2009, 81(6), 2378-2382.

(42) Ohno, Y.; Maehashi, K.; Yamashiro, Y.; Matsumoto, K. Nano Lett. 2009, 9(9), 3318-22.

(43) Alwarappan, S.; Erdem, A.; Liu, C.; Li, C. Z. J. Phys. Chem. C 2009, 113 (20), 8853-8857. 